\title{
APRENDIZAJE COLABORATIVO Y TECNOLOGÍAS DE LA INFORMACIÓN Y LA COMUNICACIÓN
}

"Para que la civilización sobreviva, debemos perfeccionar al máximo la ciencia de las relaciones humanas: la capacidad de todos los pueblos, sin distinciones, para vivir juntos y en paz en el mismo mundo."

Franklin Delano Rooselvet.

\section{María Eugenia Calzadilla}

Universidad Pedagógica Experimental Libertador, Venezuela

La sociedad actual gestiona a nivel mundial el paso a un nuevo tiempo, aún en definición; a nivel nacional se plantean cambios significativos, que más allá de la diatriba política, tocan cuestiones de fondo, por lo que es imprescindible concretar un verdadero interés por superar los conflictos y resolver los problemas que complican la concertación y el necesario consenso en torno a asuntos fundamentales para el definitivo arranque del país en pos de su desarrollo; de allí la necesidad de poseer una sana percepción emocional que permita la ponderación y el equilibrio, indispensables para la constitución de equipos de trabajo eficientes.

Esto no se aprende por dictado, ni correspondencia y forma parte de un código que el individuo va asumiendo a lo largo de su vida, que debe ser apoyado por el aparato educativo nacional, pues sólo así se podrá dar cumplimiento a los mandatos constitucionales que invocan la felicidad del colectivo.

Desde el ingreso del niño al preescolar y hasta su último grado académico, debe sentirse que la esencia de todo proceso educativo es capacitar al individuo para la convivencia y autorrealización y que en estos importantes logros las emociones lejos de ser algo que avergüence o moleste, facilitan el tránsito feliz en el camino hacia la coronación de las metas.

Es difícil comprender el cambio brusco que en términos de relaciones sociales ha dado la sociedad y su incidencia en los procesos educativos; asimismo, el impacto emocional que estos cambios han tenido sobre la humanidad, ha conducido a la sucesión de hechos antes inconcebibles como el desenfreno de la delincuencia infantil y juvenil; lo más preocupante es que allí se encuentra el futuro de la humanidad y si eso es lo que vendrá, hay que trabajar para evitarlo.

Las emociones juegan un papel decisivo al momento de realizar tareas complejas que requieren tiempo y esfuerzo. Por ello, el gerente debe saber manejar la inteligencia emocional, entendida como un conjunto de habilidades que abarquen la capacidad de motivarse y persistir frente a las decepciones, controlar el impulso y demostrar la gratificación, regular el mal humor, mostrar empatía hacia las demás personas y autoconocer un 
sentimiento cuando se presenta, variables de tipo psicológico que pudieran obstaculizar el desempeño personal y laboral, así como el logro de colaboración dentro de los equipos de trabajo. Es así como el elemento emocional resulta determinante al introducir innovaciones, particularmente de tipo tecnológico, por la constante adaptación al cambio que implican y la competencia descarnada que pueden desatar.

Los cambios fundamentales en los conceptos e ideas y la forma en que se utilicen, mueven las sociedades de una etapa a otra, por tanto es necesario considerar cognitivamente los problemas socioeconómicos como un todo y se debe conseguir que la actitud mental hacia ellos sean parte integrante del enfoque de los mismos. De ello dependerá cuál de las cuatro actitudes mentales hacia la solución y hacia el futuro esté dominando: reactiva, inactiva, proactiva o interactiva.

Los recursos humanos son elementos claves en cualquier proceso de cambio y en el aumento de la productividad y competitividad de las organizaciones. Es por ello que el gerente del nuevo milenio debe ser un individuo de grandes dotes humanas, capaz de transmitir a los subordinados esa energía activa que estimule en todos un cambio paradigmático, para unir esfuerzos e implementar estrategias efectivas frente a la crisis sociopolítica que afecta al país. En un mundo en el que el coeficiente intelectual ya no es suficiente para dirigir con eficiencia a las personas, la comprensión del papel que juega la inteligencia emocional en las organizaciones se erige como el eslabón que facilitará el logro de la excelencia en el trabajo.

El docente, desde la escuela, necesita abrirse a nuevas experiencias que actualicen su repertorio pedagógico, logrando transformar la experiencia educativa en impacto trascendente para la efectiva inserción social del individuo, en términos de sus capacidades y aptitudes para la convivencia y la autorrealización personal, profesional y laboral.

Hay que cambiar los estilos gerenciales y el clima organizacional; estimular la innovación y la flexibilización dialógicas, automotivantes y holísticas. En este orden de ideas Covey (1997) plantea que:

«los paradigmas» se emplean por lo general con el sentido de modelo, teoría, percepción, supuestos o marco de referencia; un cambio de paradigma es cuando se rompe con la tradición, con los viejos modos de pensar, con los antiguos paradigmas, ese cambio de paradigma nos empuja de modo instantáneo o gradual, que pasemos de una manera de ver el mundo a otra. Ese cambio genera poderosas transformaciones. (p. 40)

En la mayoría de las instituciones educativas venezolanas se observa la implementación de una cultura reactiva, en donde la toma de decisiones se hace de forma unilateral, producto de los patrones de conductas adoptados de viejos esquemas y modos de hacer las cosas. Con relación a este punto Hofstede, citado por Granell (1997) define que la cultura es:

Una programación colectiva de la mente que distingue a los integrantes de un grupo o categorías de otros, tiende a ser común en personas expuestas a una misma educación y experiencias de vida. Esta programación mental incluye pensamientos y sentimientos que, se entienden, han sido construidos a través de instituciones como la familia, la escuela, las asociaciones y hasta el estado. Es decir, la programación se elabora en los ambientes sociales en los que ha crecido y 
vivido. Esto es lo que se entiende generalmente como cultura nacional. Pero la cultura nacional no se queda en la mente de los individuos si no que se lleva a las organizaciones. La cultura del trabajador es una extensión de la conducta adquirida en la escuela y la familia. La conducta de un gerente es una extensión de sus experiencias familiares y escolares, al igual que espejo de la conducta de sus supervisores (p. 5).

Existe incidencia de la inteligencia emocional en el comportamiento de la labor del individuo en las organizaciones, y que ellas influyen de manera significativa en las relaciones interpersonales para crear un clima que active la creatividad y den apertura a escenarios de participación en la toma de decisiones que impulsen la mejor gestión como producto de un trabajo en equipo sinérgico.

La combinación de las estrategias ofrecidas por la inteligencia emocional y el aprendizaje colaborativo pudiera optimizar el proceso de incorporación tecnológica a la formación docente, con la finalidad de superar las barreras emocionales que pudieran existir para afrontar la alfabetización tecnológica del recurso humano, componente esencial del proceso.

\section{APRENDIZAJE COLABORATIVO Y CONSTRUCTIVISMO}

El aprendizaje colaborativo se sustenta en teorías cognoscitivas. Para Piaget hay cuatro factores que inciden e intervienen en la modificación de estructuras cognoscitivas: la maduración, la experiencia, el equilibrio y la transmisión social. Todos ellos se pueden propiciar a través de ambientes colaborativos. En la teoría constructivista (Vigotsky, 1974), el aprendiz requiere la acción de un agente mediador para acceder a la zona de desarrollo próximo, éste será responsable de ir tendiendo un andamiaje que proporcione seguridad y permita que aquél se apropie del conocimiento y lo transfiera a su propio entorno. En cuanto a las implicaciones educativas de los anterior, Coll y Solé (1990, p. 332), definen a la enseñanza como «un proceso continuo de negociación de significados, de establecimiento de contextos mentales compartidos, fruto y plataforma, a su vez, del proceso de negociación», lo que permite verificar las conexiones entre aprendizaje, interacción y cooperación: los individuos que intervienen en un proceso de aprendizaje, se afectan mutuamente, intercambian proyectos y expectativas y replantean un proyecto mutuo, que los conduzca al logro mutuo de un nuevo nivel de conocimiento y satisfacción.

El aprendizaje colaborativo, es otro de los postulados constructivistas que parte de concebir a la educación como proceso de socioconstrucción que permite conocer las diferentes perspectivas para abordar un determinado problema, desarrollar tolerancia en torno a la diversidad y pericia para reelaborar una alternativa conjunta. Los entornos de aprendizaje constructivista se definen como «un lugar donde los alumnos deben trabajar juntos, ayudándose unos a otros, usando una variedad de instrumentos y recursos informativos que permitan la búsqueda de los objetivos de aprendizaje y actividades para la solución de problemas» (Wilson, 1995, p. 27). 
El aprendizaje colaborativo es eficiente para insertar la educación dentro del proyecto de vida y conectar la evolución personal con el desarrollo de un proyecto de país coherente que favorezca la cohesión y la visión sistémica de elementos hoy fragmentados, como son: formación, educación, familia, sociedad, desempeño laboral y evolución nacional. Se estimula con este tipo de estrategia la desaparición de observadores pasivos y receptores repetitivos, superando los tradicionales hábitos de memorización utilitaria, para promover procesos dialógicos que conduzcan a la confrontación de múltiples perspectivas y a la negociación propias de la dinamicidad de todo aprendizaje que conduzca al desarrollo.

En cuanto al conocimiento, el constructivismo plantea que su valor no es absoluto, pues éste es el producto de las múltiples interpretaciones que hacen los individuos de su entorno, de acuerdo a las posibilidades de cada uno para interactuar y reflexionar. Los sujetos negocian significados a partir de la observación y valoración de aspectos de la realidad que les son comunes. «Los alumnos desarrollan su propias estrategias de aprendizaje, señalan sus objetivos y metas, al mismo tiempo que se responsabilizan de qué y cómo aprender. La función del profesor es apoyar las decisiones del alumno» (Gros, 1997, p. 99).

\section{EL MODELO DE APRENDIZAJE COLABORATIVO}

La educación tradicional, favorecida por los modelos sociopolíticos convencionales, lejos de favorecer el proceso antes descrito, se ha empeñado en exaltar los logros individuales y la competencia, por encima del trabajo en equipo y la colaboración; esta realidad, tal como lo señala Díaz Barriga (1999, p. 52-53) se evidencia «no sólo en el currículo, el trabajo en clase y la evaluación, sino en el pensamiento y la acción del docente y sus alumnos».

Si bien el conductismo planteaba la absoluta dependencia del docente, quien dominaba la situación educativa y regía en el aula sobre sus alumnos, al hablar de aprendizaje colaborativo no traspasamos esta situación al grupo, su esencia es mucho más compleja y enriquecedora: en el aprendizaje colaborativo cada participante asume su propio ritmo y potencialidades, impregnando la actividad de autonomía, pero cada uno comprende la necesidad de aportar lo mejor de sí al grupo para lograr un resultado sinérgico, al que ninguno accedería por sus propios medios; se logra así una relación de interdependencia que favorece los procesos individuales de crecimiento y desarrollo, las relaciones interpersonales y la productividad.

Los trabajos en grupo han sido práctica convencional en los diferentes niveles y modalidades del sistema educativo, ello no implica que sea ésta una práctica verdaderamente cooperativa, en la que el producto es el producto innovador de la sinergia divergente de un equipo de personas sobre un tema específico. Del grupo al equipo, hay un tránsito, cuyo valor agregado es la cooperación, pero muchas veces los mismos docentes desconocen cómo producir este logro y orientar las actividades de aprendizaje en esa dirección. 
Algunas pautas para producir aprendizaje colaborativo son: a) estudio pormenorizado de capacidades, deficiencias y posibilidades de los miembros del equipo; b) establecimiento de metas conjuntas, que incorporen las metas individuales; c) elaboración de un plan de acción, con responsabilidades específicas y encuentros para la evaluación del proceso; d) chequeo permanente del progreso del equipo, a nivel individual y grupal; e) cuidado de las relaciones socioafectivas, a partir del sentido de pertenencia, respeto mutuo y la solidaridad, y f) discusiones progresivas en torno al producto final.

Evidentemente este tipo de aprendizaje dialógico facilita el desarrollo de aquellos procesos cognitivos, como la observación, el análisis, la capacidad de síntesis, el seguir instrucciones, comparar, clasificar, tomar decisiones y resolver problemas, en los que la interacción enriquece los resultados y estimula la creatividad.

Por otra parte, el aprender en forma colaborativa permite al individuo recibir retroalimentación y conocer mejor su propio ritmo y estilo de aprendizaje, lo que facilita la aplicación de estrategias metacognitivas para regular el desempeño y optimizar el rendimiento; por otra parte este tipo de aprendizaje incrementa la motivación, pues genera en los individuos fuertes sentimientos de pertenencia y cohesión, a través de la identificación de metas comunes y atribuciones compartidas, lo que le permite sentirse "parte de», estimulando su productividad y responsabilidad, lo que incidirá directamente en su autoestima y desarrollo.

Según Díaz Barriga ( 1999) el aprendizaje colaborativo se caracteriza por la igualdad que debe tener cada individuo en el proceso de aprendizaje y la mutualidad, entendida como la conexión, profundidad y bidireccionalidad que alcance la experiencia, siendo ésta una variable en función del nivel de competitividad existente, la distribución de responsabilidades, la planificación conjunta y el intercambio de roles.

Resulta importante resaltar la necesidad de comprender el verdadero significado del aprendizaje colaborativo, pues de lo contrario se corre el riesgo de promover experiencias caracterizadas por actitudes individualistas, en las que prevalecen los conflictos, frustraciones y complejos de los miembros del grupo y no se logra entablar una interacción favorable, que conduzca a la interdependencia positiva. Atendiendo el proceso desde la conformación misma del equipo, se producirá un aprendizaje que además de resultar rico en cuanto a los productos cognoscitivos logrados, a nivel interpersonal e intrapersonal se modela y aprende valoración y responsabilidad hacia el proceso educativo, capacidad para conformar equipos de trabajo productivo y respeto por los demás y su trabajo.

En este punto, resulta importante tomar en cuenta que todo proceso grupal debe partir por la aceptación legítima de cada integrante, lograr niveles aceptables de comunicación y confianza, que permitan dar y recibir apoyo y resolver asertivamente los conflictos que de continuo se presentan en las relaciones humanas, para poder tomar decisiones conjuntas que favorezcan la consolidación como equipo, la mayoría de los docentes al iniciar un trabajo de aprendizaje con un grupo de estudiantes, tienden a repetir la repartición de contenidos en grupos elegidos aleatoriamente, cuyos productos de aprendizaje se presentan en una jornada maratónica de 
exposiciones en la que rotafolios, transparencias y video-veam ofrecen en apretados y minúsculos textos una síntesis del trabajo que van leyendo a una audiencia pasiva que se abstiene de hacer preguntas.

Este tipo de experiencias no ejerce mediación en el proceso de aprendizaje, ni se hace seguimiento e intervención al proceso de desenvolvimiento del grupo, o se aplica evaluación formativa, conociendo tanto los logros tanto colectivos como personales, o generando una reflexión seria por parte del grupo y produciendo reconocimientos que recompensen objetivamente las acciones valiosas. Es verdad que el trabajo en equipos con enfoque colaborativo requiere mayor dedicación y es mucho más meticuloso, pero produce en los estudiantes verdadero crecimiento intelectual y socioafectivo y en el docente, continua mejoría en su capacidad mediadora.

Existen al menos tres formas de poner en práctica el aprendizaje colaborativo: la interacción de pares, el tutoreo de pares y el grupo colaborativo (Tudge, 1994). La diferencia entre ellos está determinada por la igualdad en los niveles de rendimiento que exista entre los integrantes. La interación de pares consiste en la integración de grupos con participantes de diferentes niveles de habilidad, que acometen las ejecuciones en forma organizada y conjunta, participando el docente como mediador y catalizador en las experiencias de aprendizaje del grupo.

El tutoreo de pares (Tudge, 1994), involucra a estudiantes en los que se ha detectado mayor habilidad y a los que se les ha dado un entrenamiento previo para servir de coach de sus compañeros de menor nivel, mientras desempeñan el trabajo en forma conjunta; por lo general la interacción entre los estudiantes es tan fluida que logra elevar el nivel de los aprendices y consolidar el que tienen los avanzados, quienes querrán conservar su posición de adelantados y continuarán profundizando en el conocimiento.

Los grupos colaborativos por su parte, tienen mayor tamaño que los primeros y vinculan aprendices de distinto nivel de habilidad, género y procedencia; acumulan el puntaje en forma individual y grupal a lo largo de todo el período, lo que estimula la interdependencia y asegura la preocupación de todos por el aprendizaje de todos, pues el éxito colectivo depende del éxito individual. En este caso el docente debe ser más que un mediador, propiciando un proceso grupal efectivo (Johnson y Johnson, 1992, y Vásquez, Johnson y Johnson, 1993). El grupo puede ser estable o permanente, inestable o circunstancial y de base, que es aquel que va más allá del ámbito académico, desarrollando actividades de soporte y apoyo para el desarrollo integral de sus integrantes.

El aprendizaje colaborativo ha demostrado eficiencia en la superación de actitudes negativas, incrementar la motivación y el autoconcepto; por otra parte las experiencias de interacción cooperativa permiten producir un aprendizaje vinculado al entorno social del individuo, dado que propician la creación de ambientes estimulantes y participativos, en los que los individuos se sienten apoyados y en confianza para consolidar su propio estilo de aprendizaje. 
Eggen y Kauchak (1999) señalan que los estudiantes que explican y elaboran, aprenden más que los que solamente escuchan explicaciones, quienes a su vez aprenden más, que los estudiantes que aprenden solos. «El aprendizaje colaborativo alienta la elaboración, pidiendo a los estudiantes que hablen acerca de sus nuevas ideas con otros estudiantes de su grupo» (p. 301).

\section{NUEVAS TECNOLOGÍAS Y APRENDIZAJE COLABORATIVO}

El desarrollo de las nuevas tecnologías y su utilización en el proceso educativo, requiere del soporte que proporciona el aprendizaje colaborativo, para optimizar su intervención y generar verdaderos ambientes de aprendizaje que promuevan el desarrollo integral de los aprendices y sus múltiples capacidades; en este orden de ideas Ruíz y Ríos (1990) señalan la conveniencia del enfoque Aprendizaje asistido por el Computador (CAL), contrapuesto al de Instrucción asistida por el Computador (CAI), que promueve la transmisión de información su posterior comprobación y tiende a propiciar la sustitución del docente; el Aprendizaje asistido por el Computador, con énfasis en lo cognoscitivo, enriquece el papel del docente, poniendo a su disposición los elementos que conjugará según su pericia para la puesta en escena en la que el aprendiz será el protagonista, alcanzando una actitud favorable hacia la superación de errores, dada la continua exposición a estimulantes experiencias que conllevan nuevos retos y requieren el desarrollo de nuevas habilidades, destrezas y conocimientos.

Cabe destacar que para promover el verdadero logro de experiencias de aprendizaje colaborativo, se debe partir por la constitución de pequeños grupos, entre dos y cuatro integrantes; por otra parte el lapso durante el cual se dará el trabajo conjunto, también interviene en el logro, pues aquellos que prolongan la duración de las sesiones de trabajo, tendrán oportunidad de conocerse mejor e integrarse efectivamente para generar aprendizaje, así como el desarrollo de las habilidades sociales para su exitosa inserción en el grupo.

En este plano, las tecnologías también benefician el logro de aprendizaje colaborativo, pues para poder aprovechar las bondades del equipo computarizado, así como la comprensión y el aprendizaje, es recomendable un máximo de tres personas trabajando en un equipo. Una vez concluida la sesión presencial, el trabajo en equipo puede verse prolongado mediante los diferentes recursos tecnológicos: chat, correo, listas o foros, proporcionan la oportunidad de nuevos intercambios.

Pueden producirse experiencias positivas de aprendizaje cuando los alumnos comparten sus descubrimientos, se brindan apoyo para resolver problemas y trabajan en proyectos conjuntos. Por otra parte esta tecnología interactiva permite desarrollar, extender y profundizar las habilidades interpersonales y penetra las barreras culturales a medida que estudiantes y docentes aprenden a comunicarse mediante las nuevas formas que propone este medio. 
Desde el punto de vista pedagógico, las TICs representan ventajas para el proceso de aprendizaje colaborativo, en cuanto a:

a) Estimular la comunicación interpersonal, que es uno de los pilares fundamentales dentro de los entornos de aprendizaje virtual, pues posibilita el intercambio de información y el diálogo y discusión entre todas las personas implicadas en el proceso. En función del diseño del curso, existen herramientas que integran diferentes aplicaciones de comunicación interpersonal o herramientas de comunicación ya existentes (como el correo electrónico o el chat). Estas aplicaciones pueden ser síncronas, como la audio/videoconferencia, las pizarras electrónicas o los espacios virtuales y asíncronas como los foros o listas de discusión.

b) Las nuevas tecnologías facilitan el trabajo colaborativo, al permitir que los aprendices compartan información, trabajen con documentos conjuntos y faciliten la solución de problemas y toma de decisiones. Algunas utilidades específicas de las herramientas tecnológicas para el aprendizaje cooperativa son: transferencia de ficheros, aplicaciones compartidas, asignación de tareas, calendarios, chat, convocatoria de reuniones, lluvia de ideas, mapas conceptuales, navegación compartida, notas, pizarra compartida, votaciones, etc.

c) Seguimiento del progreso del grupo, a nivel individual y colectivo; esta información puede venir a través de los resultados de ejercicios y trabajos, test de autoevaluación y coevaluación, estadística de los itinerarios seguidos en los materiales de aprendizaje, participación de los estudiantes a través de herramientas de comunicación, número de veces que han accedido estos al sistema, tiempo invertido en cada sesión y otros indicadores que se generan automáticamente y que el docente podrá chequear para ponderar el trabajo de cada grupo, pero a su vez los estudiantes podrán también visualizar el trabajo que tanrto ellos como el resto de los grupos han efectuado y aplicar a tiempo correctivos y estrategias metacognitivas que tiendan a remediar un desempeño inadecuado.

d) Acceso a información y contenidos de aprendizaje: mediante las bases de datos on line o bibliográficas, sistemas de información orientados al objeto, libros electrónicos, publicaciones en red, centros de interés, enciclopedias, hipermedias, simulaciones y prácticas tutoriales que permiten a los estudiantes intercambiar direcciones, diversificar recursos e integrar perspectivas múltiples.

e) Gestión y administración de los alumnos: permite el acceso a toda aquella información vinculada con el expediente del estudiante e información adicional, que le pueda ser útil al docente en un momento dado, para la integración de grupos o para facilitar su desarrollo y consolidación.

f) Creación de ejercicios de evaluación y autoevaluación, con los que el docente podrá conocer el nivel de logro y rediseñar la experiencia de acuerdo a su ritmo y nivel y al estudiante le ofrecerán retroalimentación sobre el nivel de desempeño.

En la medida en que se van identificando nuevas competencias relevantes para el mundo de la información y el conocimiento en el que vivimos, van surgiendo nuevos modelos formativos, que en sus concreciones tendrán que adaptarse a las exigencias, requerimientos y oportunidades que la evolución tecnológica representa; la formación interactiva on line, amplía los maestros disponibles y las informaciones y experiencias para compartir, lo que por otra vía resultaría imposible. 
Las TICs propician una postura de flexibilidad cognitiva, pues cada usuario puede establecer itinerarios particulares y recorrerlos según su gusto y necesidad: textos, proyectos, propuestas, experiencias, nuevos medios para la interacción y el trabajo con los aprendices y docentes conocidos cara a cara o con otros remotos e invisibles, enriquecen el proceso de aprendizaje y abren la voluntad de cooperar que en la presencialidad quizás permanecería pasiva, cubierta por el temor de hablar o el miedo escénico de interactuar en un grupo que no siempre tiene tolerancia y receptividad hacia todos sus miembros por igual.

De allí que las principales ventajas derivadas del uso de estrategias de aprendizaje colaborativo, derivan en el desarrollo y mejora continua de las competencias del docente para ejercer el apoyo y acompañamiento responsables y creativos y en cuanto al estudiante, el desarrollo de estrategias de relación social, metacognición y metaevaluación, lo que le confiere mayor autonomía y pertinencia a sus participaciones.

Pese al gran entusiasmo por adaptar los procesos educativos a los medios de interacción virtual, cabe señalar que al evaluar su eficiencia en términos de la educación y crecimiento emocional de individuos y grupos de trabajo, es mucho lo que aún aporta la presencialidad. Los grupos de aprendizaje no van a volverse colaborativos tan sólo por estar en la red. Es necesario identificar, evaluar y aumentar los recursos emocionales y las aptitudes sociales de los integrantes de cada grupo y del grupo como tal y esto se logra definiendo y modelando valores que impacten el desarrollo humano de los aprendices. La mejor propuesta formativa será en todo caso, aquella que pueda conjugar cada estrategia de la forma más conveniente y en su justa dimensión, sin abusar o subestimar su uso y sin olvidar que el fin educativo, que es el bienestar social y el desarrollo debe prevalecer y orientar cualquier acción educativa que se emprenda.

\section{BIBLIOGRAFÍA}

CAMPBEL, y otros (2000): Inteligencias múltiples. Usos prácticos para la enseñanza y el aprendizaje. Editorial troquel (Argentina).

COLL, y SOLÉ (1990): «La interacción profesor/alumno en el proceso de enseñanza y aprendizaje», en C. Coll; J. Palacios, y A. Marchesi (eds.): Desarrollo psicológico y educación II. Madrid, Alianza editorial.

COVEY (1997): El liderazgo centrado en principios. Madrid, Editorial Paidos.

DÍAZ BARRIGA (1999): Estrategias docentes para un aprendizaje significativo. Una interpretación constructivista. México, Mcgraw-Hill.

EGGEN, y KAUCHAK (1999): Estrategias docentes. Enseñanza de contenidos curriculares y desarrollo de habilidades de pensamiento. Brasil, Fondo de cultura económica.

GRANeLl (1997): La formación de recursos humanos de alto nivel en Venezuela. Estado, empresa y academia. Papeles de trabajo IESA, n. ${ }^{\circ} 26$. Caracas, Ediciones IESA.

Gros, B. (1997): Diseño y programas educativos. Pautas pedagógicas para la elaboración de software. Barcelona, Editorial Ariel. 
JONHSON, y JONHSON (1992): Cooperative learning increasing. Washinton D.C., College Faculty, ERIC Digest.

Ruíz, y Ríos (1990): «El uso de la informática en la educación», en: Investigación y Postgrado, Vol. 5 n. 2 (pp. 59-89).

Ríos (1999): «El constructivismo en educación», en: Laurus. Año 5. n8. pp.16-23.

Tudge (1994): Vigotsky: la zona de desarrollo próximo y su colaboración en la práctica de aula. Nueva York, Universidad de Cambridge.

VÁSQUEZ; JONHSON, y JONHSON (1993): «The impact of cooperative learning om the performance and retention of US Navy Air Traffic Controller Trainees», en: The Journal of Social Psichology, 133 (6), pp. 769-783.

VIGOTSKY (1979): El desarrollo de los procesos psicológicos superiores. Madrid, Editorial Grijalbo.

WiLSON (1995): Cómo valorar la calidad de la enseñanza. Madrid, Paidos. 


\title{
Contactar
}

Revista lberoamericana de Educación

\author{
Principal OEI
}

